\title{
Power Control in Spectrum Sharing Systems with Almost-Zero Inter-System Signaling Overhead
}

\author{
Mohammad G. Khoshkholgh \\ Carleton University \\ ghadir@sce.carleton.ca
}

\author{
Halim Yanikomeroglu \\ Carleton University \\ halim@sce.carleton.ca
}

\begin{abstract}
Power allocation in spectrum sharing systems is challenging due to excessive interference that the secondary system could impose on the primary system. Therefore, an interference threshold constraint is considered to regulate the secondary system's activity. However, the primary receivers should measure the interference and inform the secondary users accordingly. These cause design complexities, e.g., due to transceiver's hardware impairments, and impose a substantial signaling overhead. We set our main goal to mitigate these requirements in order to make the spectrum sharing systems practically feasible. To cope with the lack of a model we develop a coexisting deep reinforcement learning approach for continuous power allocation in both systems. Importantly, via our solution, the two systems allocate power merely based on geographical location of their users. Moreover, the inter-system signalling requirement is reduced to exchanging only the number of primary users that their QoS requirements are violated. We observe that compared to a centralized agent that allocates power based on full (accurate) channel information, our solution is more robust and strictly guarantees $\mathrm{QoS}$ requirements of the primary users. This implies that both systems can operate simultaneously with almost-zero inter-system signaling overhead.
\end{abstract}

\section{INTRODUCTION}

Spectrum sharing among coexisting services is a powerful solution for coping with the never-ending increase of traffic demands, the scarcity of spectrum, and chronic poor spectral efficiency [1]. Under 4G/5G the notion of cognitive heterogenous networks (HetNets) have been investigated in which the primary system, which has higher priority in spectrum access, shares the spectrum with the secondary systems. Many of the developed resource allocation and networking solutions require levels of interactions between coexisting services, strong modelling assumptions regarding the occupancy of the spectrum, availability of (full) channel state information (CSI) between coexisting systems, which, in turn, renders staggering complexity and imposes substantial signaling overhead [2], [3]. Accordingly, the feasibility of spectrum sharing for practical scenarios becomes hard to argue for and has inspired recent research activities [4]. This is transpired (partially) as a results of the emerge of deep reinforcement learning (DRL) [5] and machine learning (ML) [6] techniques.

Two common spectrum sharing methods are underlay and overlay [1], [3], where in the former the secondary service is allowed to access the spectrum as long as the service degradation of the primary system stays in the permissible zone. The

This work was supported by Huawei Canada Co., Ltd. latter requires spectrum sensing in order to mitigate crosssystem interferences altogether. Our focus is on the underlay paradigm, thus the need for spectrum sensing is eliminated. Conventionally, an interference threshold constraint (ITC) is imposed to manage the harmful activity of the secondary users [1], [3], which requires the primary users to measure interference and feedback it along with corresponding CSI to the secondary service, which 1) requires substantial modifications of the primary service, 2) increases the complexity particularly under the impaired hardware [7] and intra-system interference, and 3) leads to high signalling overhead. We attempt to develop power control solution with minimum upgrade of the primary system operation, robust to lack of channel information, and with minimum required inter-system signaling overhead.

1) Literature Review: Reference [8] uses deep Q-learning for user selection in underlay secondary massive MIMO system when the inter-system CSI is not available. In [9], the authors use deep Q-learning for passively predicting the used modulation of the primary service in order to adjust the secondary users' transmit powers. However, the approach is only applicable for one primary link, and poses substantial computation overhead on the secondary system. Reference [10] uses tabular Q-learning along with the use of radio environment map to guide the secondary service for more effectively protecting the primary service's QoS. In [11], deep Q-learning is used to empower the secondary transmitter to manage its harvesting energy and spectrum access. Only one primary transceiver and one secondary transceiver is assumed.

2) Our Contributions: One drawback of deep Q-learning solutions is that one should firstly quantizes the transmission powers into a fixed number of bins, which could lead to the curse of dimensionality by growing the number of users [12]. Instead, we focus on continuous DRL solutions based on actor-critic structure [13], [14]. In particular, we adopt deep policy gradient DRL algorithm of proximal policy optimization (PPO) [15] to learn continuous power allocation merely based on geographical location information of users.

Note that in the literature the focus is usually on either the primary service or the secondary service. In contrast, we focus on both systems. In effect, we consider those scenarios that both systems act as independent intelligent agents but with minimal information exchange. The primary service is only informing the secondary service with the number of 
its users that their rate requirements (QoS) are violated. Our experiments show that the developed solution is able to fulfill the QoS requirement of the primary system strictly, even more effectively than the centralized solution (learning power allocation in both systems at a central agent). This implies that both systems can operate simultaneously with almost-zero inter-system signaling overhead. Further, our solution tends to allocate higher power and harness higher data rate across systems compared to the centralized solution. Importantly, we observe that allocating power solely based on geographical locations of users does not cast any performance lost compared to the case that full CSI is available. Finally, our solution incorporates the transceiver hardware impairments [7]. The impact of such prevalent impairments are usually ignored under conventional solutions due to complexity of modelling and the induced mathematical intractability, which due to the model-free nature of our approach is straightforward to incorporate.

\section{System ModeL}

We focus on the interference channel power control problem consisting of two coexisting (non-cooperative and noncompetitive) systems. The first system, which has a higher service priority, is denoted by primary system and the second system is referred to by the secondary system which can be subject to penalties if violating regulatory requirements for spectrum access. Both systems use the same spectrum. The secondary system must be vigilant regarding the QoS degradation of the primary service.

The primary (secondary) system consists of $K_{p}$ (resp. $K_{s}$ ) single-antenna transceivers pairs. Each transmitter has its own intended receiver. Let us denote $h_{k k}^{p p} \in \mathbb{R}^{+}$(resp. $h_{k k}^{s s}$ ) the direct channel power gain between primary (resp. secondary) transmitter $k$ and primary (resp. secondary) receiver $k$. The communication of all transmitters $k$ interferes with other communication channels (primary and secondary) through channel power gains $h_{k j}^{p p}$ (from primary transmitter $k$ to primary receiver $j$ ), $h_{k j}^{p s}$ (from primary transmitter $k$ to secondary receiver $j$ ), $h_{k j}^{s p}$ (from secondary transmitter $k$ to primary receiver $j$ ), $h_{k j}^{s s}$ (from secondary transmitter $k$ to secondary receiver $j$ ). We assume the interference is considered as noise in both systems. On the other hand, transceivers suffer from hardware impairment [7]. The impairment is mainly a function of two parameters $\kappa_{t} \in[0.08,0.175]$ and $\kappa_{r} \in[0.08,0.175]$ sanding as the distortion level at the transmitter and the receiver which are measured in error vector magnitudes (EVMs). Similar to [7], the signal distortion at the secondary receiver $k$ can be modelled as complex Gaussian random variable with power

$$
D_{k}^{s}=\left(\kappa_{r}^{s}\right)^{2} P_{k}^{s}+\left(\kappa_{t}^{s}\right)^{2} \sum_{j} h_{j k}^{s s} P_{j}^{s}+\left(\kappa_{t}^{s}\right)^{2} \sum_{j} h_{j k}^{p s} P_{j}^{p} .
$$

Likewise,

$$
D_{k}^{p}=\left(\kappa_{r}^{p}\right)^{2} P_{k}^{p}+\left(\kappa_{t}^{p}\right)^{2} \sum_{j} h_{j k}^{p p} P_{j}^{p}+\left(\kappa_{t}^{s}\right)^{2} \sum_{j} h_{j k}^{s p} P_{j}^{s} .
$$

As seen, the power of the distortion noise at the receivers is a function of transmission powers at all the transmitters. Therefore, the experienced signal-to-interference-plus-noiseand-distortion ratio (SINDR) at the primary receiver $k$ and secondary receiver $k$, respectively, is

$$
\begin{aligned}
\operatorname{SINDR}_{k}^{p} & =\frac{h_{k k}^{p p} P_{k}^{p}}{\sigma_{k}^{2}+D_{k}^{p}+\sum_{j \neq k} h_{j k}^{p p} P_{j}^{p}+\sum_{j} h_{j k}^{s p} P_{j}^{s}}, \\
\operatorname{SINDR}_{k}^{s} & =\frac{h_{k k}^{s s} P_{k}^{s}}{\sigma_{k}^{2}+D_{k}^{s}+\sum_{j \neq k} h_{j k}^{s s} P_{j}^{s}+\sum_{j} h_{j k}^{p s} P_{j}^{p}},
\end{aligned}
$$

where $D_{k}^{p}$ accounts for the collective impact of transceiver impairments due to the distortion noises, $\sigma_{k}^{2}$ is the noise power at the receiver $k$ and $P_{k}^{p} \in\left[0, \hat{P}_{k}^{p}\right]$ is the (continuous) transmission power of the primary transmitter $k$, which should be smaller than the maximum permissible transmission power $\hat{P}_{k}^{p}$. The same holds true at the secondary system. As a result, the SINDRs at the primary and secondary systems are very intricate functions of the transmission powers. The data rate of the primary (secondary) user $k$ is calculated by the Shannon's formula $r_{k}^{p}=\log \left(1+\operatorname{SINDR}_{k}^{p}\right.$ ) (resp. $\left.r_{k}^{s}=\log \left(1+\operatorname{SINDR}_{k}^{s}\right)\right)$.

The QoS requirement of the primary users are specified through the data rate $r_{t h}$. The resource allocation problem in the primary system can be specified via the following optimization problem:

$$
\mathcal{O}_{p}: \max _{0 \leq P_{k} \leq \hat{P}_{k}^{p}, \forall k} \sum_{k=1}^{K}\left(r_{k}^{p}-r_{t h}\right) .
$$

The secondary system is designed for maximizing the sum energy efficiency (EE) subject to the QoS requirement of the primary system. Note that the primary service does not measure the interference imposed by the secondary system as it poses substantial complexity mainly due to interference from primary users as well as the distortion noises. In effect, the primary system only provides the secondary system with

$$
\mathrm{nQoS}_{p}=\sum_{k} \mathrm{NACK}_{k}^{p}
$$

where $\operatorname{NACK}_{k}^{p} \in\{0,1\}$. Here, $\operatorname{NACK}_{k}^{p}=0$ means $r_{k}^{p} \geq r_{t h}$. Thus, $\mathrm{nQoS}_{p}$ is the sum of the number of primary users that their service requirements are violated. Note that the amount of signaling between the primary and secondary systems is at most $\left\lfloor\log K_{p}\right\rfloor$ bits, which is much lower than the case that CSI needs to be feeded-back. The secondary system should solve the following optimization problem:

$$
\mathcal{O}_{s}: \max _{0 \leq P_{j}^{s} \leq \hat{P}_{j}^{s}} \sum_{j=1}^{J} \frac{r_{j}^{s}}{\tau\left(P_{j}^{s}+P_{0}^{s}\right)+P_{j}^{d}\left(r_{j}^{s}\right)} \text {, s.t. } \operatorname{nQoS}_{p}=0 .
$$

As seen, in formulating the EE we include the decoding energy $P_{j}^{d}\left(r_{j}^{s}\right)$ at the receivers too [16], which is usually ignored in the literature, as it is hard to mathematically formulate and usually renders substantial intractability. The decoding energy is generally a function of transmitted data rate. 
Comparing $\mathcal{O}_{s}$ with $\mathcal{O}_{p}$, we note that the secondary service is subject to a strict QoS requirement of primary service, which aims at maximizing $r_{k}^{p}-r_{t h}$ of its users. This vividly demonstrates the priority of services, and permits us measuring how effective the secondary service is in obliging with spectrum sharing rules.

Solving $\mathcal{O}_{s}$ with $\mathcal{O}_{p}$ is complex due to complex nature of QoS constraint at the secondary service and unknown sources of hardware impairments. Our goal here to develop power allocation strategies at both primary and secondary services that are robust against lack of model. To these ends, we utilize DRL framework. Note also that to minimize the complexity of CSI acquisition we pursue the scenarios that only geographical location information of the transceivers in each system is available, which is not shared across systems.

\section{BACKGROUND}

In continuous DRL an agent, operating in an uncertain environment with the continuous state and action spaces, interacts with the environment in a sequential style to learn an optimal policy [12]. In each interaction the agent takes an action $\boldsymbol{a}_{t} \in \mathbb{R}^{B}$ ( $B$ is the action dimension) based on its observation of the environment state $s_{t} \in \mathbb{R}^{S}$ ( $S$ is the dimension of the state space), which leads the agent to the new state $s_{t+1}$ upon on collecting the bounded reward $r_{t} \in \mathbb{R}$. The policy guides the agent to what action should be taken in a certain state in order to maximize the reward via maximizing the discounted expected reward $J(\pi)=\mathbb{E}_{\pi} \sum_{t} \gamma^{t} r_{t}\left(\boldsymbol{s}_{t}, \boldsymbol{a}_{t}\right)$ by finding an optimal policy $\pi$ [5]. Parameter $\gamma \in(0,1]$ is the discount factor prioritizing short-term rewards and the expectation is on the policy $\pi$. Advantage function $A_{\pi}\left(\boldsymbol{s}_{t}, \boldsymbol{a}_{t}\right)$ (or simply $A_{\pi}$ ) is the subtraction of the Q-function and statevalue function, i.e., $A_{\pi}=Q_{\pi}\left(\boldsymbol{s}_{t}, \boldsymbol{a}_{t}\right)-V_{\pi}\left(\boldsymbol{s}_{t}\right)$. It measures the relative advantage value of action $\boldsymbol{a}_{t}$.

In DRL the policy is approximated via high-capacity DNN parameterized by $\boldsymbol{\theta}$, i.e., $\pi_{\boldsymbol{\theta}}\left(\boldsymbol{a}_{t} \mid \boldsymbol{s}_{t}\right)$ (or for short $\pi_{\boldsymbol{\theta}}$ ). We focus on stochastic policies by which the DNN deterministically maps the state to a vector that specifies a distribution over the action space, i.e., $\boldsymbol{a}_{t} \sim \pi_{\boldsymbol{\theta}}$. To learn $\pi_{\boldsymbol{\theta}}$ we use policy gradient methods: [5]

$$
\nabla_{\boldsymbol{\theta}} J(\boldsymbol{\theta})=\boldsymbol{g}=\mathbb{E}_{\pi_{\boldsymbol{\theta}}} \sum_{t} \nabla_{\boldsymbol{\theta}} \log \pi_{\boldsymbol{\theta}}\left(\boldsymbol{a}_{t} \mid \boldsymbol{s}_{t}\right) A_{\boldsymbol{\theta}}\left(\boldsymbol{s}_{t}, \boldsymbol{a}_{t}\right) .
$$

In practice the above expectation should be estimated over a batch of data collected under the current policy via Monte Carlo (MC) technique (sample based estimate of the policy gradient) $\sqrt{2}$. The agent iteratively collects data $\left(\boldsymbol{s}_{t}, \boldsymbol{a}_{t}, r_{t}, \boldsymbol{s}_{t+1}\right)$ by interacting with the environment via policy $\pi_{\boldsymbol{\theta}}$, estimates the gradient of the policy, updates the policy, and then discards the data. This is basically the policy gradient of REINFORCE

\footnotetext{
${ }^{1}$ For policy $\pi$, the state-value function $V_{\pi}\left(s_{t}\right)$ measures the expected discounted reward from state $\boldsymbol{s}_{t}$ via $V_{\pi}\left(\boldsymbol{s}_{t}\right)=$

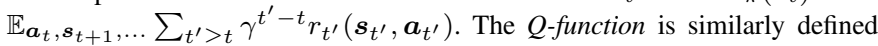

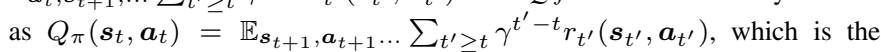
state-value function for a given action.

${ }^{2}$ We use symbol $\hat{x}$ as the MC estimation of quantity $x$.
}

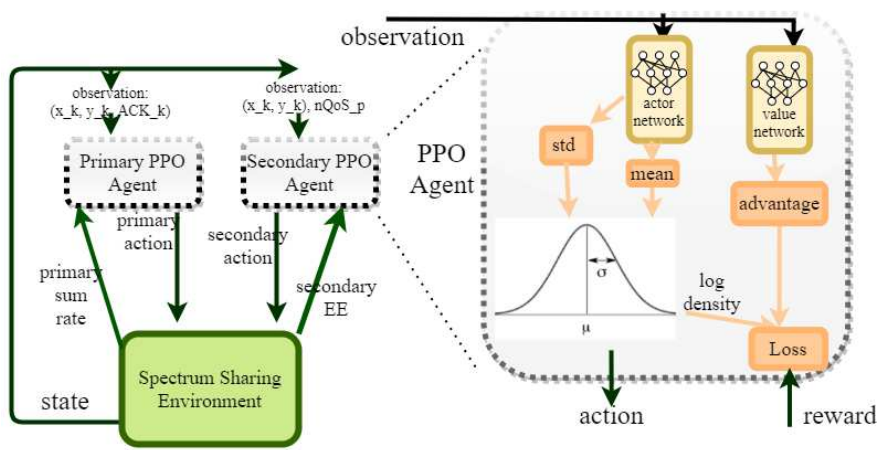

Fig. 1. Coexisting PPO agents for power control in spectrum sharing systems.

algorithm, which is also known as vanilla policy gradient (VPG). However, VPG algorithm is not sample efficient, is brittle in convergence, and suffers from high variance.

\section{Coexisting PPO Agents}

\section{A. Actor-Critic Policy Gradient}

Besides learning the policy it is recommended to learn a value function, which helps reducing the variance of gradient, thus stabilizes VPG algorithm [17]. This is the core idea of actor-critic technique in which a DNN_called the actor or the policy net-updates the policy while another DNN_called the critic or the value net-updates the value function's parameters denoted by $\omega$.

To better understand actor-critic structure refer to Fig. 1. As seen from PPO agent's structure, the agent's observation is feed to both policy and value networks. From the value network the advantage value is estimated. The policy network provides a distribution over the action in continuous dimension. It is customary to choose an expressive distribution such as multivariate Gaussian distribution. The output of the policy network calculates the mean value of this distribution. We do not need to accommodate a separate output for calculating the standard deviation as it is calculated from the heads of the policy network. This approach is known to substantially stabilize the learning procedure of the policy network. As seen from the illustration, by knowing the distribution the agent produces actions via sampling. The agent should also calculate the logarithm of the distribution in order to calculate the loss function, which also requires the value of advantage and reward that is received from environment. The loss function is then used to update both value and policy networks as is discussed in details in what follows.

\section{B. Proximal Policy Optimization (PPO)}

Mathematically, under PPO algorithm the target policy is derived by solving the following optimization problem [15]:

$$
\operatorname{Maximize}_{\boldsymbol{\theta}} \mathbb{E}_{\pi_{\boldsymbol{\theta}_{k}}}\left[\min \left\{\frac{\pi_{\boldsymbol{\theta}}(\boldsymbol{a} \mid \boldsymbol{s})}{\pi_{\boldsymbol{\theta}_{k}}(\boldsymbol{a} \mid \boldsymbol{s})} A_{\boldsymbol{\theta}_{k}}, c\left(\epsilon, A_{\boldsymbol{\theta}_{k}}\right)\right\}\right],
$$

where $\epsilon \in(0,1]$ is the clip ratio and $c\left(\epsilon, A_{\boldsymbol{\theta}_{k}}\right)=(1+$ $\left.\operatorname{sgn}\left(A_{\boldsymbol{\theta}_{k}}\right) \epsilon\right) A_{\boldsymbol{\theta}_{k}}$ where $\operatorname{sgn}($.$) is the sign operator. Usually,$ 
it is sufficient to set the value of $\epsilon$ around 0.1 , which is the case of our experiments. Under this objective, if the advantage function is positive the objective function reduces to $\min \left(1+\epsilon, \frac{\pi_{\boldsymbol{\theta}}(\boldsymbol{a} \mid \boldsymbol{s})}{\pi_{\boldsymbol{\theta}_{k}}(\boldsymbol{a} \mid \boldsymbol{s})}\right) A_{\boldsymbol{\theta}_{k}}$, which is increasing if action becomes more likely (meaning $\pi_{\boldsymbol{\theta}}(\boldsymbol{a} \mid \boldsymbol{s})$ increases). Nonetheless, to ensure stability, it is not desirable to increase the policy far from the current policy $\pi_{\boldsymbol{\theta}_{k}}(\boldsymbol{a} \mid \boldsymbol{s})$, which is regulated via threshold $1+\epsilon$. On the other hand, if the advantage function is negative, the objective function reduces to $\max \left(1-\epsilon, \frac{\pi_{\boldsymbol{\theta}}(\boldsymbol{a} \mid \boldsymbol{s})}{\pi_{\boldsymbol{\theta}_{k}}(\boldsymbol{a} \mid \boldsymbol{s})}\right) A_{\boldsymbol{\theta}_{k}}$. Hence, to improve the objective the action should be taken to decrease $\pi_{\boldsymbol{\theta}}(\boldsymbol{a} \mid \boldsymbol{s})$, meaning it needs to become less likely. Again, it is not desirable to diverge too much from the current policy hence the reason to impose the limit $1-\epsilon$.

In Algorithm 1 we provide the required steps to update the policy and value networks. PPO algorithm has an outer loop indexed by $l=1,2, \ldots, L$. For each iteration $l$, the policy is fixed, letting the agent take actions and collect new bach of data. The iteration comprises of an inner loop indexed by $n$ with length $N$ (the number of transitions which also known as batch size), each of which associated with an episode with length $T$. Using the collected transitions the advantage function is estimated via MC technique, which are used to update the policy network and value network.

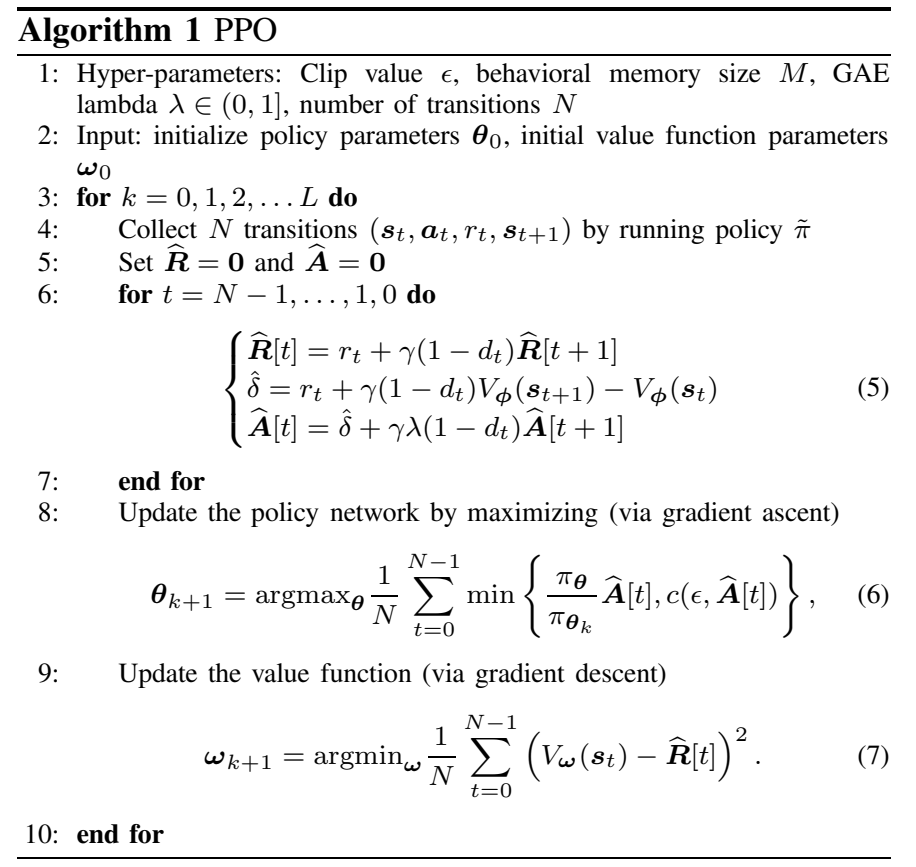

Updating Policy: Updating policy is based on solving optimization problem $\tilde{\mathcal{O}}$ which is done in several steps (Step 5 to Step 8). First, we need to estimate $\sqrt{3}$ the rewards-to-go $\widehat{\boldsymbol{R}}$ and advantages $\widehat{\boldsymbol{A}}$. In the calculation of the advantages $\widehat{\boldsymbol{A}}$ we adopt the generalized advantage estimation (GAE) [17] where $\lambda \in(0,1]$ is a given parameter to improve the stability.

\footnotetext{
${ }^{3}$ In [5, $d_{t} \in\{0,1\}$, where $d_{t}=1$ implies that the episode is terminated As a result, the reward of the terminated time step of the episode is not included in calculation of the advantages and rewards-to-go.
}

Value Network: The update of the value network $V_{\boldsymbol{\omega}_{k}}$ is done in Step 9. Using the rewards-to-go $\widehat{\boldsymbol{R}}$ the value network is updated by mean-squared-error regression.

\section{Coexisting PPO Agents}

Our solution to power allocation in the considered spectrum sharing system is based on coexisting PPO agents-one agent for power control at the primary service and another one for the power control at the secondary service (see Fig. 11). Both agents are responsible for taking their own (optimal) actions. The reward in the primary agent is defined as

$$
r_{p}=\left\{\begin{array}{ll}
0.1\left(\sum_{k}\left(r_{k}^{p}-r_{t h}\right)\right)-5 \Delta_{p} & \Delta_{p}>0 \\
\sum_{k}\left(r_{k}^{p}-r_{t h}\right) & \Delta_{p}=0
\end{array},\right.
$$

where $r_{k}^{p}$ is the rate of primary user $k, \Delta_{p}$ is the penalty defined as the sum of excessive power (related to the lower and upper limits of allowable transmission power at the primary system) that the policy is allocated. Hence, the reward attempts to teach the agent to stick to allowable power range. Furthermore, the reward encourages the agent to assign power to achieve the transmission rate higher than the rate threshold. Note that when $\Delta_{p}>0$ we scale down the reward $\sum_{k}\left(r_{k}^{p}-r_{t h}\right)$ to send a signal to the agent that though the power was not respective to the boundaries but it was constructive (with accordance to the value of $r_{k}^{p}-r_{t h}$ ). Similarly, for the secondary agent the reward, which is related to the sum of EE, is defined as

$$
r_{s}=\left\{\begin{array}{ll}
0.1 \sum_{k} e e_{k}^{s}-2 \mathrm{nQoS}_{p}-5 \Delta_{s} & \Delta_{s}>0 \\
\sum_{k} e e_{k}^{s}-10 \mathrm{nQoS}_{s} & \Delta_{s}=0
\end{array},\right.
$$

where $e e_{k}^{s}$ is the EE of secondary user $k$, and $\mathrm{nQoS}_{p}$ is the number of primary users with violated QoS requirement. Again, the reward is constructed to teach the agent the power boundaries.

Note that the environment's state is the full CSI between all transmitters and all receivers, along with the transmission rate of primary users, EE of secondary users, and nQoS ${ }_{p}$. But, each agent has its own observation of the environment. The primary service's observation, which is used to train its associated PPO agent (see Fig. 1), includes the geographical locations of its users along with the transmitted data rate. Also, the secondary service's observation, which is used to train its agent (see Fig. 1), is the geographical location of its users along with the value of $\mathrm{nQoS}_{p}$ and the EE of its users. Both agents attempt to learn their optimal policy through their observations and achieved rewards. Therefore, no knowledge of full CSI, hardware impairments, and decoding model at the secondary system is assumed. Also, besides minimum information exchange between agent, each agent is basically unaware of other agent's policy.

\section{EXPERIMENTS}

For the experiments we use the pytorch library [18]. For each experiment we consider 6 different random seeds and calculate the average values accordingly. In our experiment 


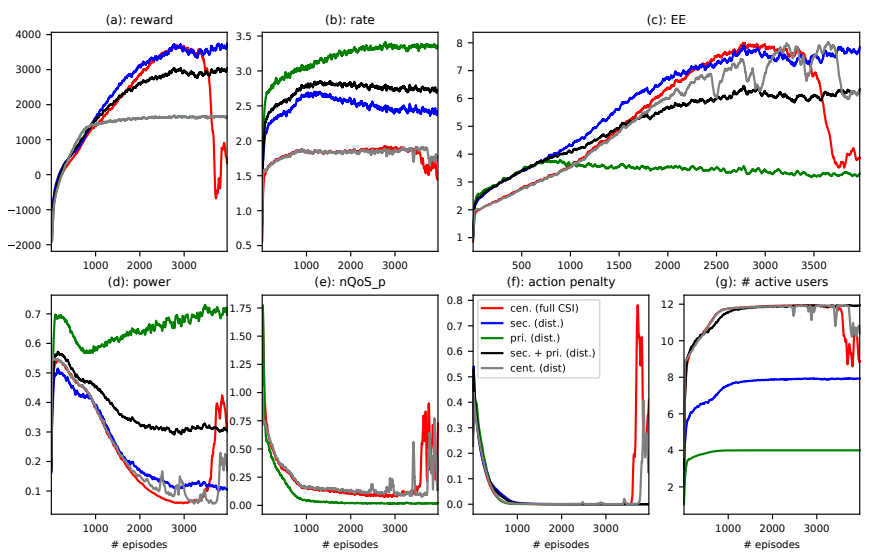

Fig. 2. Results of $\operatorname{Ex} 1\left(K_{s}=8\right.$ and $\left.K_{p}=4\right)$.

we set $L=4000, N=500$ with episode length $T=500$. Furthermore, we set $\gamma=0.1, \epsilon=0.1$, and GAE $\lambda=0.94$.

\section{A. Configuration}

1) Channel Model: We consider a circular area with radius $100 \mathrm{~m}$, and randomly locate users in it. The wireless channel is based on 3GPP Line-of-Sight (LOS)/non-LOS (NLOS) pathloss model [19], whereby $L_{j k}=\left\|X_{j k}\right\|^{-\alpha_{l}} \sim p_{l}\left(\left\|X_{j k}\right\|\right)$ for $l \in\{L, N\}$ in which $p_{\mathrm{N}}\left(\left\|X_{j k}\right\|\right)=1-p_{\mathrm{L}}\left(\left\|X_{j k}\right\|\right)$ is the probability of LOS that is a function of distance $\left\|X_{j k}\right\|$ : $p_{\mathrm{L}}\left(\left\|X_{j k}\right\|\right)=\min \left\{\frac{D_{0}}{\left\|X_{j k}\right\|}, 1\right\}\left(1-e^{-\frac{\left\|X_{j k}\right\|}{D_{1}}}\right)+e^{-\frac{\left\|X_{j k}\right\|}{D_{1}}}$. Also, $\alpha_{\mathrm{L}}$ (resp. $\left.\alpha_{\mathrm{N}}\right)$ is the path-loss exponent associated with LOS (resp. NLOS) component where $\alpha_{\mathrm{N}}>\alpha_{\mathrm{L}} . D_{1}$ and $D_{0}$ are hyper-parameters which can take different values for different environments. We set the channel parameters as $\alpha_{L}=2.4$, $\alpha_{N}=3.78, D_{0}=18 \mathrm{~m}, D_{1}=36 \mathrm{~m}$, and the background noise power $-173 \mathrm{dBm} / \mathrm{Hz}$. The fading power gain under the LOS mode is modelled by Nakagami-m distribution with parameter $m=10$. Under the NLOS mode the fading is modelled via unit-mean exponential random variable. We also consider large-scale shadowing with mean zero $\mathrm{dB}$ and standard deviation $5 \mathrm{~dB}$ under LOS mode and $8.6 \mathrm{~dB}$ under NLOS mode. Receivers and transmitters are allowed to dislocate by up to 5 meters in a random direction at the start of each iteration. However, we are making sure that the receivers stay in the simulation area. The distortion levels at the transmitters and receivers are $\kappa_{t}=\kappa_{r}=0.1$. Also, the rate threshold at the primary service is $r_{t h}=0.5(\mathrm{bit} / \mathrm{sec} / \mathrm{Hz})$.

2) Policy and Value Networks: Policy of primary agent is modelled stochastically as a multivariate Normal distribution with diagonal covariance matrix. The mean of this distribution is a DNN with 3 dens layers. The first and second layers are with input/output dimensions $S_{p} / 64$ and $64 / 64$ respectively, where $S_{p}$ is the primary agent's observation space dimension. This DNN has two heads, one for the mean value and the other for the logarithm of the standard deviation. Each of these are modelled by its associated dense layer with size $64 / B$ where $K_{p}$ is the action dimension (number of primary users). Similarly, the value net is also a DNN with three layers with the difference that the last layer has dimensions $64 / 1$. The activation functions are Tanh [6]. The observation space of the

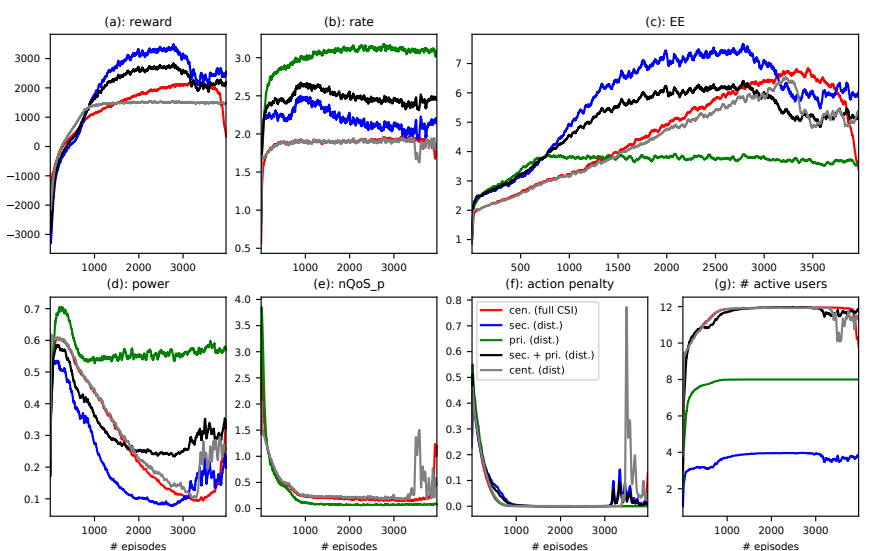

Fig. 3. Results of $\operatorname{Ex} 2\left(K_{s}=4\right.$ and $\left.K_{p}=8\right)$.

primary agent as the stacked 2-D distance between transceivers of the primary users and their transmission rates, hence $S_{p}=$ $\left(K_{p}^{2}+K_{p}\right)$. In the following, we refer to this agent by pri.(dist).

The above configuration stays correct for the secondary agent after adjusting for its observation space dimension $S_{s}$ and action space dimension $B_{s}=K_{s}$. The observation space of the secondary agent is as the stacked 2-D distance between transceivers of the secondary users, their EE, and $\mathrm{nQoS}_{p}$. Thus, $S_{s}=\left(K_{s}^{2}+K_{s}+1\right)$. We refer to this agent by sec.(dist) in the following.

\section{B. Objectives}

We here consider two experiments: Ex1 in which $K_{s}=8$ and $K_{p}=4$ and Ex2 in which $K_{s}=4$ and $K_{p}=8$. Results of Ex1 and Ex2 are shown in Fig. 2 and Fig. 3, respectively. In our experiments we are interested to investigate

- Q1: Do agents learn from reward signals?

- Q2: Are the agents capable of learning the power control boundaries?

- Q3: Whether the secondary service is able to adhere to the QoS requirement of the primary service or not?

- Q4: How much performance is gained/lost via using two separate agents compared to a centralized agent that obtain the power in both systems?

- Q5: How the lack of full CSI affects the performance?

To answer item Q4 we consider a PPO agent that receives state which includes full CSI across all transceivers in the network and allocates power in both systems. Thus, state space has dimension $S=\left(\left(K_{s}+K_{p}\right)^{2}+K_{s}+K_{p}+1\right)$ and the action space has dimension $B=K_{s}+K_{p}$. We denote this agent by cent.(full CSI). On the other hand, to answer item Q5 we use the same setup as the cent.(full CSI) agent but instead of full CSI we use only geographical locations of all users. We call this agent cent.(dist). Note that we properly scale and sum the results of pri.(dist) and sec.(dist) agents to compare the collective performance of the coexisting scenario with cent.(full CSI) and cent.(dist) agents.

\section{Results}

1) Q1: As seen from Fig. 22-(a) and Fig. 3-(a) the pri.(dist) agent keeps improving its reward until it is reaching the 
plateau, which is because of the rate threshold of the primary service. Recall that we set $r_{t h}=0.5$, while the pri.(dist) is able to guarantee data rate $3.5(\mathrm{bit} / \mathrm{sec} / \mathrm{Hz})$ which is $2.5(\mathrm{bit} / \mathrm{sec} / \mathrm{Hz})$ higher than the threshold $\left(4 \times r_{t h}=2\right)$. We also note that the pri.(dist) agent gains higher rate compared to sec.(dist) agent as the latter's objective is to maximize its EE. In effect, from Fig. 2-(c) and Fig. 3-(c) the sec.(dist) gains much higher EE compared to pri.(dist) agent. Importantly, we note from Fig. 2-(d) and Fig. 3-(d) the pri.(dist) agent allocates much higher power than the sec.(dist), which demonstrates that agents are able to understand the implications of their actions: lower power shall be allocated to improve the EE. Note that both agents tend to keep all the users active (see Fig. 2-(g) and Fig. 3. $(\mathrm{g}))$.

2) Q2: As seen from Fig. 2.(f) and Fig. 3-(f) under both experiments the agents are able to learn the action boundaries very quickly. However, centralized agents may violate the boundaries occasionally.

3) Q3: As seen from Fig. 2.(e) and Fig. 3-(e) under both experiments the sec.(dist) agent is learned the QoS requirement of the primary service very effectively. Interestingly, under the coexisting scenario the sec.(dist) agent is more effective than the centralized agents. The practical implications of this experiment is that the signaling overhead between the primary and secondary systems can reach to almost-zero as the secondary service can learn very quickly to respect to QoS requirement of the primary system. As a result, under our solution, both systems can operate almost independent of each other.

4) Q4: By examining Fig. 2 and Fig. 3 we note that generally the coexisting agents show higher stability compared to the centralized ones. This could be due to much higher state space of the latter and also the fact that the reward becomes more complex to efficiently learn from. In effect, we observe that the aggregate impact of coexisting agents results in higher transmission rates compared to the centralized ones. We further note from Fig. 2.(d) and Fig. 3-(d) that the coexisting agents tend to allocate more power compared to the centralized ones. However, regarding EE, the superiority of the coexisting agents agent the centralized ones is not that clear-cut and could be affected depending on the number of users in each system.

5) Q4: Finally, to understand the impact of CSI, we compare the performance of cent.(full CSI) and cent.(dist) agents. As seen from Fig. 2 and Fig. 3 in general the lack of CSI does not cast any particular effect on reward, rate, EE, transmission power, and active users. This is an important finding noticing the related practical implications, e.g., much lower signaling overhead and transmitter/receiver complexities.

\section{CONCLUSIONS}

We developed coexisting power allocator agents for spectrum sharing systems without imposing a hard interference threshold at the primary service. The developed power allocation circumvented the inter-system signalling overhead into exchanging an integer number from the primary system to the secondary system, standing for the number of primary users that their QoS requirements are violated, which was shown to be almost-zero. Our solution further did not required the knowledge of path-loss, shadowing, and fading, so that the two systems allocate power entirely independently merely based on geographical location of their corresponding transceivers. We observed that compared to the centralized system that allocates power based on the accurate CSI our solution is more robust and can guarantee QoS requirements of the primary users more strictly.

\section{REFERENCES}

[1] A. Goldsmith, S. A. Jafar, I. Maric, and S. Srinivasa, "Breaking spectrum gridlock with cognitive radios: An information theoretic perspective," Proceedings of the IEEE, vol. 97, no. 5, pp. 894-914, May 2009.

[2] Y. Wu, Q. Zhu, J. Huang, and D. H., "Revenue sharing based reshource allocation for dynamic spectrum access networks," IEEE J. Sel. Areas. Commun., vol. 32, no. 11, pp. 2280-2296, Nov. 2014.

[3] M. G. Khoshkholgh, K. Navaie, and H. Yanikomeroglu, "Access strategies for spectrum sharing in fading environment: Overlay, underlay, and mixed," IEEE Trans. Mobile Comput., vol. 9, no. 12, pp. 1780-1793, Dec. 2010.

[4] Z. Qin, X. Zhou, L. Zhang, Y. Gao, Y.-C. Liang, and G. Y. Li, "20 years of evolution from cognitive to intelligent communications," IEEE Trans. Cognitive Commun. and Net., vol. 6, no. 1, pp. 6-20, Mar. 2020.

[5] R. S. Sutton and A. G. Barto, Reinforcement Learning: An Introduction. MIT Press, Cambridge, 2017.

[6] I. Goodfellow, Y. Bengio, and A. Courville, Deep Learning. MIT Press, Cambridge, 2016.

[7] E. Bjornson, M. Matthaiou, and M. Debbah, "A new look at dual-hop relaying: performance limits with hardware impairments," IEEE Trans. Commun., vol. 61, no. 11, pp. 4512-4525, Nov. 2013.

[8] Z. Shi, X. Xie, and H. Lu, "Deep reinforcement learning based intelligent user selection in massive MIMO underlay cognitive radios," IEEE Access, vol. 7, pp. 110884-110 894, Aug. 2019.

[9] F. S. Mohammadi and A. Kwasinski, "Neural network cognitive engine for autonomous and distributed underlay dynamic spectrum access," arXiv:1806.11038, 2018.

[10] N.Morozs, T. Clarke, and D. Grace, "Heuristically accelerated reinforcement learning for dynamic secondary spectrum sharing," IEEE Access, vol. 3, pp. 2771-2783, Dec. 2015.

[11] K. Pathak and A. Banerjee, "Harvest-or-transmit policy for cognitive radio networks: A learning theoretic approach," arXiv:1906.00548, 2019.

[12] T. Lillicrap, J. Hunt, A. Pritzel, N. Heess, T. Erez, Y. Tassa, D. Silver, and D. Wierstra, "Continuous control with deep reinforcement learning," International Conference on Learning Representations (ICLR), May 2016.

[13] Y. Duan, X. Chen, R. Houthooft, J. Schulman, and P. Abbeel, "Benchmarking deep reinforcement learning for continuous control," International Conference on Machine Learning (ICML), vol. 48, pp. 1329-1338, Jun. 2016.

[14] C. Qiu, Y. Hu, Y. Chen, and B. Zeng, "Deep deterministic policy gradient (ddpg)-based energy harvesting wireless communications," IEEE Internet of Things Journal, vol. 6, no. 5, pp. 8577-8588, Oct. 2019.

[15] J. Schulman, F. Wolski, P. Dhariwal, A. Radford, and O. Klimov, "Proximal policy optimization algorithms," arXiv:1707.06347, 2017.

[16] A. Arafa and S. Ulukus, "Optimal policies for wireless networks with energy harvesting transmitters and receivers: effects of decoding costs," IEEE J. Sel. Areas. Commun., vol. 33, no. 12, pp. 2611-2625, Dec. 2015.

[17] J. Schulman, P. Moritz, S. Levine, M. Jordan, and P. Abbeel, "Highdimensional continuous control using generalized advantage estimation," International Conference on Learning Representations (ICLR), May 2016.

[18] A. Paszke, S. Gross, S. Chintala, G. Chanan, E. Yang, Z. DeVito, Z. Lin, A. Desmaison, L. Antiga, and A. Lerer, "Automatic differentiation in pytorch,' in Workshop on Neural Information Processing Systems (NIPSW), 2017.

[19] 3GPP, "5G; study on channel model for frequencies from 0.5 to 100 GHz (version 14.0.0 Release 14). TR 38.901," Sep. 2017. 